\title{
Effect of strain rate on strength and deformation behavior of an Fe-Mn-Al-Ni shape memory alloy
}

\author{
Sebastian Henschel ${ }^{1, *}$ and Lutz Krüger ${ }^{1}$ \\ ${ }^{1}$ Institute of Materials Engineering, TU Bergakademie Freiberg, Gustav-Zeuner-Str. 5, 09599 Freiberg, \\ Germany
}

\begin{abstract}
The strength and deformation behavior of an Fe-Mn-Al-Ni shape memory alloy at different strain rates was studied. Furthermore, the effect of grain size was investigated. To this end, a batch with bamboo-like grain arrangement and a batch with smaller, nevertheless coarse, grains were analyzed. Tensile tests at quasi-static, intermediate, and dynamic loading rates were performed. Digital image correlation and microstructural analysis revealed the localized deformation and phase transformation in favorable oriented grains. At higher strain rates, a increased number of orientations was activated for the phase transformation. A higher strain rate resulted in an increased strength for the coarse-grained material while the bamboo-like material did not show this effect. The analysis of fracture surfaces revealed ductile fracture and cleavage fracture for all strain rates.
\end{abstract}

\section{Introduction}

Shape memory alloys (SMA) that exhibit pseudoelastic behavior are suitable for structural applications, e.g. as energy absorbers in an earthquake environment [1]. Fe-based shape memory alloys (Fe-SMA) offer promising advantages compared to conventional SMA, e.g. NiTi-based SMA. One obvious advantage is the lower cost of the alloying elements.

Vollmer et al. [2] investigated the pseudoelastic behavior and showed its degradation with cyclic loading. The degradation was caused by activating different martensite variants. However, the effect of the loading rate was not studied.

According to Dolce et al. [1], typical maximum oscillation frequencies of an earthquake are in the range of $1-4 \mathrm{~Hz}$. Loading from zero to maximum tensile force takes one fourth of the oscillation period, i.e. $0.06-0.25 \mathrm{~s}$, leading to strain rates of approx. $0.2-0.8 \mathrm{~s}^{-1}$. Since single bursts may also occur, the mechanical behavior at higher strain rates is also of interest.

Tseng et al. [3] and Vollmer et al. [4] showed that the temperature sensitivity of the critical stress for the transformation from austenite to martensite is relatively low in a wide temperature range $\left(-150\right.$ to $\left.300{ }^{\circ} \mathrm{C}\right)$. Hence, possible adiabatic heating at high strain rates may only have a small effect on the phase transformation.

Saletti et al. [5] investigated the martensitic phase transformation in a polycrystalline Ni$\mathrm{Ti}$ alloy at different strain rates. They observed a constant ratio of elongation of the tensile specimen to cumulated area of the transformed material. This ratio was independent of the strain rate in the range of $10^{-4}$ to $20 \mathrm{~s}^{-1}$. The area of the transformed material was measured

\footnotetext{
*e-mail: sebastian.henschel@iwt.tu-freiberg.de
} 
by means of digital image correlation (DIC) and infrared thermography. Abuzaid et al. [6] studied the temperature change during pseudoelastic deformation (elastocaloric effect) of a Fe-Mn-Al-Ni shape memory alloy. At a strain rate of $0.2 \mathrm{~s}^{-1}$, a relatively small temperature change of ca. $1 \mathrm{~K}$ was measured. Furthermore, they observed a pronounced effect of grain boundaries and crystal orientation. The result was the build-up of transformed zones at grain boundaries and the formation of cracks.

The aim of this study was to investigate the strength and deformation behavior of an Fe-Mn-Al-Ni shape memory alloy at different loading rates. To this end, tensile tests in a servohydraulic universal testing machine and an instrumented drop tower were performed. The local deformation behavior was analyzed by digital image correlation. The microstructures before and after tensile tests were studied by means of scanning electron microscopy.

\section{Materials and methods}

The chemical composition of the studied Fe-Mn-Al-Ni alloy is given in Table 1. The alloy was heat treated in two different ways. The first batch underwent a cyclic heat treatment in order to promote abnormal grain growth. Details can be found elsewhere, see [7]. Hence, a bamboo-like microstructure, with only one grain boundary in the area of interest is generated (denoted as material A). The second batch (denoted as material B) was only solution treated $\left(\mathrm{Ar}, 1225^{\circ} \mathrm{C}, 3 \mathrm{~h}\right)$ and quenched in water $\left(80^{\circ} \mathrm{C}\right)$.

The heat treatment was performed after machining (EDM) a block with the specimen contour (material A) or after machining the final specimen (material B). For material A, the block was sliced by EDM after the heat treatment in order to obtain the final tensile specimens, see Figure 1.

The initial microstructures of both materials are given in Figure 2. The specimens of material A were characterized by one grain boundary which was nearly perpendicular to the loading axis and was approximately in the center of the gauge section. Material B showed polygonal grains with sizes of about $500 \mu \mathrm{m}$. At the grain boundary, $\gamma$ phase was present. This phase is unwanted, since no further phase transformation to $\gamma^{\prime}$ can take place.

Tensile tests at different strain rates at ambient temperature were performed. For the strain rates of $10^{-3} \mathrm{~s}^{-1}$ and $10^{-1} \mathrm{~s}^{-1}$, a servohydraulic testing machine was used. An instrumented drop tower was applied for the dynamic tensile tests at $\dot{\varepsilon} \approx 10^{2} \mathrm{~s}^{-1}$. The force was derived from strain gauges (SG) at the elastically deforming part of the specimen (see Figure 1) that was calibrated with the load cell in the servohydraulic machine. Global and local strain

Table 1: Chemical composition in weight percent. Balance $=\mathrm{Fe}$.

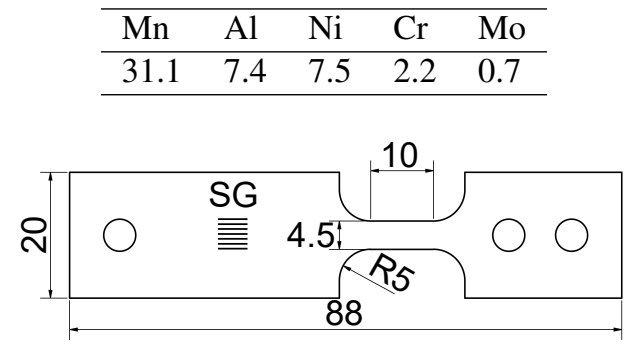

Fig. 1: Geometry of the specimens, used for all strain rates. Thickness: $2 \mathrm{~mm}$. 

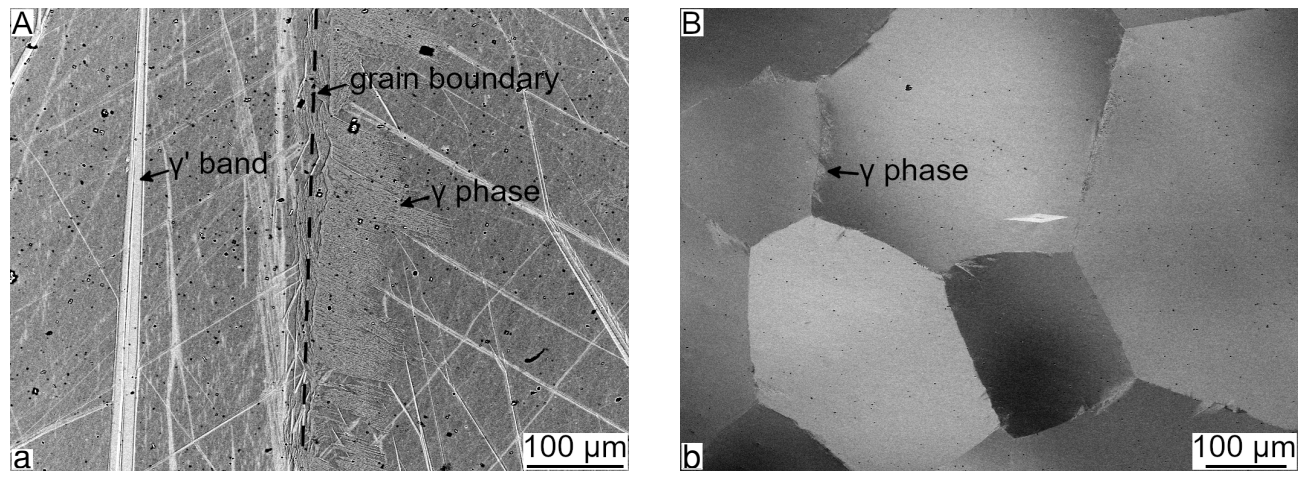

Fig. 2: Initial microstructures of materials A and B containing $\gamma$ phase at the grain boundaries and regions of transformed phase $\left(\gamma^{\prime}\right)$. The loading direction is horizontal.

analyses were performed by applying an optical extensometer (Rudolph 200XR), a low-speed camera (Canon EOS 80D) and a high-speed camera (Photron Fastcam SA-Z). The frames of the cameras were analyzed by means of digital image correlation.

Both the initial and the deformed microstructures were studied by means of scanning electron microscopy (SEM) and electron backscatter diffraction (EBSD). Consequently, the phase transformation during the loading was investigated.

\section{Results and discussion}

\subsection{Strength and deformation behavior}

Figure 3 shows stress/strain diagrams of both materials at different strain rates. Material A (Figure 3a) showed a lower stress level and less strain rate sensitivity than material B. Since there was only one grain boundary in the gauge section of the samples of material A, nearly no strengthening effect due to grain boundaries could occur.

All samples of material A had the same crystallographic orientation, i.e. approximately a [213] direction with respect to the loading axis for both grains in the gauge length. This orientation usually leads to a relatively high pseudoelastic deformation.
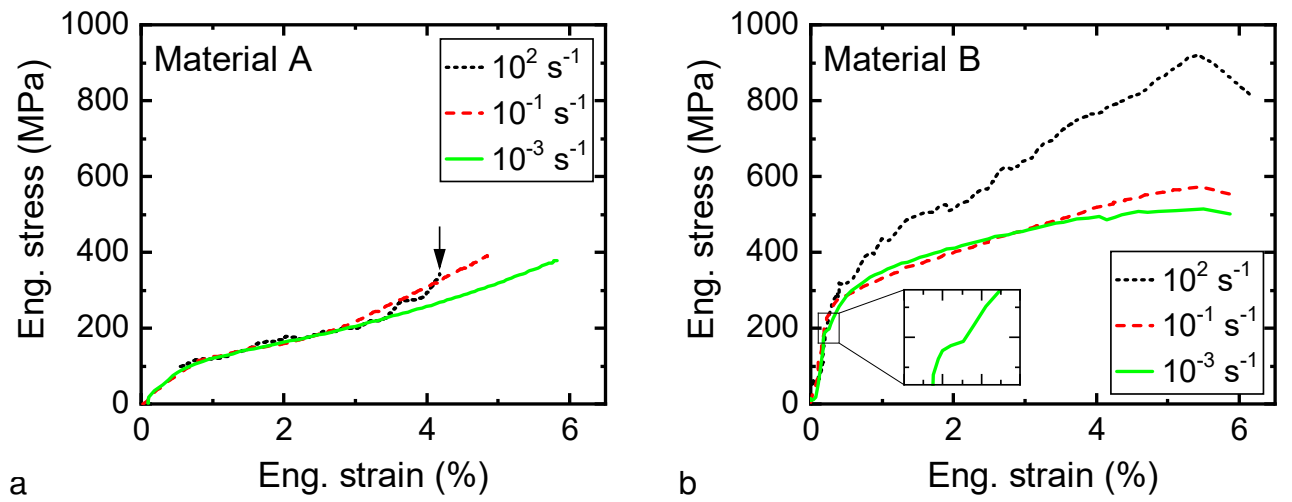

Fig. 3: Stress/strain diagrams of materials $A$ and $B$ at different strain rates 
A, $10^{-3} \mathrm{~s}^{-1}$

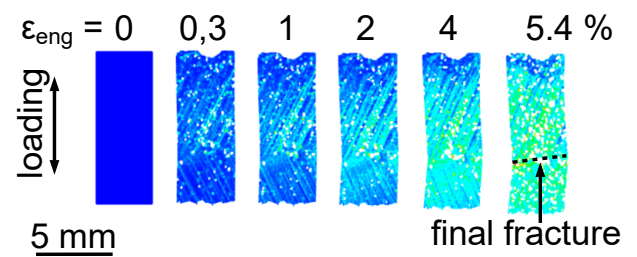
a

$$
\varepsilon_{\mathrm{vM}}=\begin{array}{llllll}
0 & 6 & 12 & 18 & 24 & 30 \%
\end{array}
$$

Fig. 4: Results of the digital image correlation for material $A$ at loading rates of $10^{-3} \mathrm{~s}^{-1}$ (a) and $10^{2} \mathrm{~s}^{-1}$ (b). $\varepsilon_{\text {eng }}$ - engineering strain, $\varepsilon_{\mathrm{vM}}$ - von Mises equivalent strain.
A, $10^{2} \mathrm{~s}^{-1}$

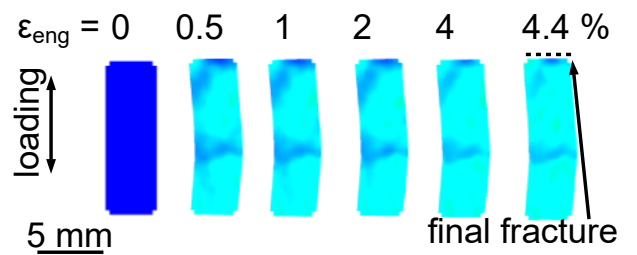

$\varepsilon_{\mathrm{vM}}=\begin{array}{llllll}0 & 6 & 12 & 18 & 24 & 30 \%\end{array}$

For material A at quasi-static loading, a stress plateau was observed at a strain interval between ca. 0.3 to $4 \%$. At higher strain rates, the stress plateau was finished at approx. $2.5 \%$ engineering strain. Taking also the local strain analysis in Figure 4 into account, the stress plateau was the result of the formation of deformation bands. The phase transformation within one grain is essentially confined to one direction.

For material B, a stress plateau was hardly noticeable. At quasi-static loading at a stress of ca. $200 \mathrm{MPa}$, an inflection point (see enlarged in Figure 3b) was observed which is attributed to pseudoelastic deformation. The pseudoelastic deformation in material A was less pronounced than in other materials with bamboo-like structure [2]. This was attributed to the missing aging treatment that produces fine precipitates. These precipitates promote the pseudoelastic behavior [8]. In material B, the phase transformation in each grain took place at different stress levels. Hence, the superposition showed no distinct stress plateau.

The force instrumentation of material A at $\dot{\varepsilon} \approx 10^{2} \mathrm{~s}^{-1}$ was only usable for a part of the test. After a strain of about $4 \%$ (see arrow in Figure 3a), the used strain gauges registered large strains due to phase transformation just below the grid of the gauges. This phenomenon shall be shortly discussed here. During the tensile tests at the highest loading rates, it is necessary to measure the axial force directly within the specimen. Hence, a part of the specimen was used that deforms linear-elastic even at force maximum, i.e. the point of ultimate tensile strength. This procedure is well established and standardized [9]. However, when testing such large-grained materials or even single crystals, a favorably oriented crystallographic plane may be present that induces inelastic deformation. Consequently, the part of the specimen that is used for force measurement has to be thicker in order to reduce the stresses. Even the most favorable oriented crystallographic plane should not be activated during plastic deformation of the gauge section. Another solution may be the separation of gauge section and "load cell" with negative consequences at high strain rate testing, like additional compliance and interfaces that lead to signal oscillations superposing the actual signal.

The tensile specimens were loaded axially. Since there was clearly no statistical grain orientation in material A, an apparent bending of the specimens was observed, see Figure 4. Hence, the grains can not deform in arbitrary directions. Consequently, there was a superposition of axial and lateral loading.

At the highest loading rate (Figure 4b), the transformation bands were not clearly visible. One obvious reason was the lower resolution of the high-speed camera. At $10^{2} \mathrm{~s}^{-1}$, a spatial resolution of ca. $50 \mathrm{px} / \mathrm{mm}$ was achieved, while the resolution was $270 \mathrm{px} / \mathrm{mm}$ at $10^{-3} \mathrm{~s}^{-1}$. However, even with that relatively low resolution, single deformation bands shall be detectable. Another reason was the less distinct formation of deformation bands with 

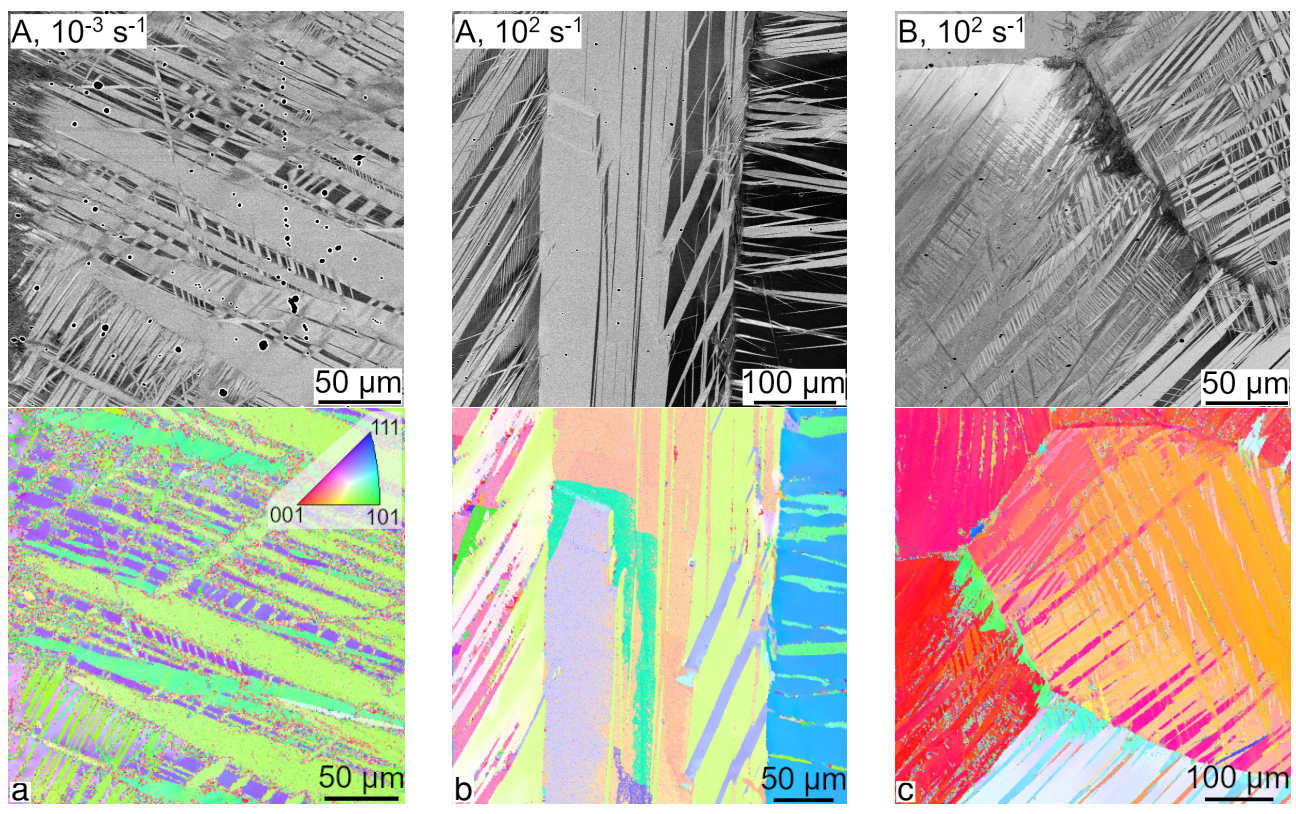

Fig. 5: Phase transformation near the fracture surfaces (distance ca. $0.5-1 \mathrm{~mm}$ ) for material $\mathrm{A}(\mathrm{a}+\mathrm{b})$ and material B (c). Upper row: BSE contrast, lower row: orientation maps (inverse pole figure is valid for all maps). The loading direction is horizontal. The orientations of the grains are given with respect to the loading axis.

only one orientation. This behavior at high strain rates was also observed by microstructural analysis, see below.

\subsection{Microstructural evolution}

The martensitic transformation from the face-centered cubic $\alpha$ phase to the body-centered cubic $\gamma^{\prime}$ phase was detected by means of SEM and EBSD. The phase transformation in material $\mathrm{A}$ at quasi-static loading rates resulted mainly in one martensite variant, see Figure 5a. Even bands with different directions showed the same crystallographic orientation. At the highest strain rate of $10^{2} \mathrm{~s}^{-1}$, see Figure $5 \mathrm{~b}$, a higher variety of martensite crystal orientations was observed. For material B (Figure 5c), also multiple orientations were activated during deformation.

The number of activated martensite variants corresponds to the stress/strain behavior shown before. Higher stresses are needed at increased strain rate to activate the transformation in unfavorable oriented bands.

\subsection{Fracture surfaces}

The fracture surface analysis of material A revealed a mixture of ductile and cleavage fracture throughout the investigated loading rates. As an example, the fracture surface is shown in Figure 6. From the investigated specimens, no trend of the ratio of ductile and cleavage fracture areas could be derived. In contrast, amount of cleavage fracture in material B increased with increasing strain rate. Furthermore, intergranular cleavage fracture was favored at the highest strain rate. 

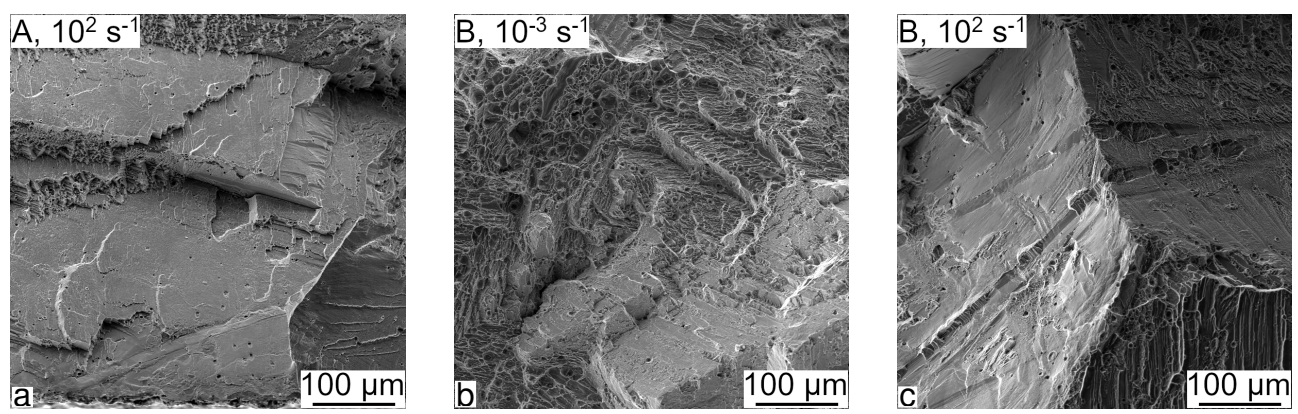

Fig. 6: Fracture surfaces of material A (a) and material B (b+c).

\section{Summary and conclusions}

The present study presents the strain rate dependent mechanical and microstructural behavior of an iron-based shape memory alloy with two different microstructures. It was shown that the stress plateau due to the pseudoelastic deformation was only detectable at the lowest strain rates. It was most noticeable at material A. Material B exhibited an increasing flow stress and approximately constant elongation at fracture with increasing strain rate. These effects were attributed to the increase of martensite fraction with increasing strain rate. Furthermore, it was shown that the martensitic phase transformation exhibits less localization during dynamic deformation. The fracture surface analysis revealed a transition from a mixture of ductile and transgranular cleavage fracture to intergranular cleavage fracture with increased loading rate.

The authors thank Dr. M. Vollmer (University of Kassel, Inst. of Materials Engineering) for providing the material, Dr. M. Kriegel (Inst. of Materials Science) and F. Fennert for support during heat treatment, C. Krancher for support during mechanical testing, and Dr. A. Weidner for discussing some results.

\section{References}

[1] M. Dolce, D. Cardone, R. Marnetto, Earthquake Engng. Struct. Dyn. 29, 945 (2000)

[2] M. Vollmer, P. Krooß, M.J. Kriegel, V. Klemm, C. Somsen, H. Ozcan, I. Karaman, A. Weidner, D. Rafaja, H. Biermann et al., Scripta Mater. 114, 156 (2016)

[3] L.W. Tseng, J. Ma, B.C. Hornbuckle, I. Karaman, G.B. Thompson, Z.P. Luo, Y.I. Chumlyakov, Acta Mater. 97, 234 (2015)

[4] M. Vollmer, P. Krooß, I. Karaman, T. Niendorf, Scripta Mater. 126, 20 (2017)

[5] D. Saletti, S. Pattofatto, H. Zhao, Mech. Mater. 65, 1 (2013)

[6] W.Z. Abuzaid, Y. Wu, R. Sidharth, F. Brenne, S. Alkan, M. Vollmer, P. Krooß, T. Niendorf, H. Sehitoglu, Shap. Mem. Superelasticity 5, 263 (2019)

[7] M. Vollmer, T. Arold, M.J. Kriegel, V. Klemm, S. Degener, J. Freudenberger, T. Niendorf, Nat. Commun. 10, 2337 (2019)

[8] P. La Roca, A. Baruj, C.E. Sobrero, J.A. Malarría, M. Sade, J. Alloy Compd. 708, 422 (2017)

[9] ISO 26203-2, Metallic materials - Tensile testing at high strain rates - Part 2: Servohydraulic and other test systems (2011) 